\title{
Haar-MRTD Time and Space Adaptive Grid Techniques for Practical RF Structures
}

\author{
Nathan Bushyager and Manos M. Tentzeris \\ School of ECE, The Georgia Institute of Technology, Atlanta, GA 30332-0250
}

\begin{abstract}
This paper presents techniques that can be used with the Haar MRTD method for time and space adaptive gridding. For the first time, absolute and relative thresholds for wavelet functions are used to change the resolution as a function of time and space. This technique is applied to the composite cell Haar MRTD scheme, which is capable of representing multiple conductors (and other subcell elements) per cell. Using this technique, complex structures can be represented more efficiently than with fixed grid techniques. Included is a comparison of the Haar method with FDTD, as well as the advantages of the Haar time-space adaptive method (10-40\% economy in memory and execution time) with fixed grid FDTD and MRTD techniques. A dual patch antenna structure suitable for RF front ends in modern multilayer substrates is presented, the final version will also include an LC CPW structure suitable for metamaterial applications.
\end{abstract}

Index Terms - MRTD, FDTD, time-domain, Haar, wavelet, adaptive grid.

\section{INTRODUCTION}

The commodization of RF and microwave components is occurring at a constantly increasing pace. As this occurs, design pressures common to digital components are placed on RF components. However, design tools for these components are still unbearably slow. While the number and quality of synthesis tools, i.e. tools that can be used to generate components based on available materials and desired parameters, are increasing, the performance of any component when used in a modern multilayer system still must be verified and optimized using a full-wave technique. By the very nature of the effects that these techniques measure, their speed cannot be easily increased. Furthermore, due to the complex geometries used in modern system-on-chip and system-on-package structures, it is not possible to use a wide variety of techniques based on analyzing a limited number of propagating modes in a structure. Thus, in the design of any modern RF system, full-wave tools are necessary and are not likely to be replaced at any time in the near future.

In order to decrease the time required to design a modern RF circuit, the full-wave tools used to model the circuit must be as efficient as possible. One technique that has been proposed is the multiresolution time-domain method (MRTD) [1]. MRTD is a general technique that is derived by expanding Maxwell's curl equations with wavelet basis functions and applying the method of moments using Galerkin's method. The advantage of this technique is that the resolution of the grid, determined by the number of wavelet coefficients used in each grid cell, can be varied as a function of both time and space.

The choice of wavelet basis functions determines many of the characteristics of the resulting scheme, such as grid size and time step requirements. For any choice of wavelet functions, there are tradeoffs involving algorithmic complexity and the number of coefficients required per wavelength. For this investigation, the Haar basis functions are chosen. Haar basis functions are the most simple wavelet functions that can be employed, but in many ways they are the most flexible. A recent paper has presented methods that can be used to simulate effects that are smaller than a cell in size using the Haar method [2]. Using this method, any structure that can be simulated in FDTD can be simulated in MRTD, with the advantage of time and space adaptive gridding.

The advantage of the MRTD time and space adaptive gridding has been mentioned in several papers [1-3]. However, the technique has not been applied to any structures up to this point. In this paper, a 3D Haar MRTD simulator is presented that uses time- and space- adaptive gridding to model a dual patch antenna structure that examines isolation between separate transmit and receive bands on a single substrate. The final paper will include a distributed LC CPW line structure that has an effective negative refractive index [4]. Both of these structures feature small elements embedded in larger circuits. It has been predicted that the time and space adaptive grid will significantly reduce execution time and memory requirements for this class of structure. These claims are evaluated in this paper.

\section{MRTD BACKGROUND}

As has been stated, the MRTD method is derived by applying the method of moments to Maxwell's curl equations. A number of papers have been written outlining this method [1-3,5-6]. A brief overview of the MRTD field update method is presented here in order to provide a framework for discussing the time and space adaptive scheme. The notation that is used here is based on that used in [5]; it has been generalized in this case to simplify the discussion of the MRTD scheme.

Maxwell's curl equations are,

$$
\nabla \times \mathbf{E}(t)=-\frac{\partial \mathbf{B}(t)}{\partial t}-\mathbf{M}(t)
$$




$$
\nabla \times \mathbf{H}(t)=\frac{\partial \mathbf{D}(t)}{\partial t}+\mathbf{J}(t)
$$

The field values in (1) and (2) can be expanded as a series of scaling functions and wavelets (in space), and pulses in time, where,

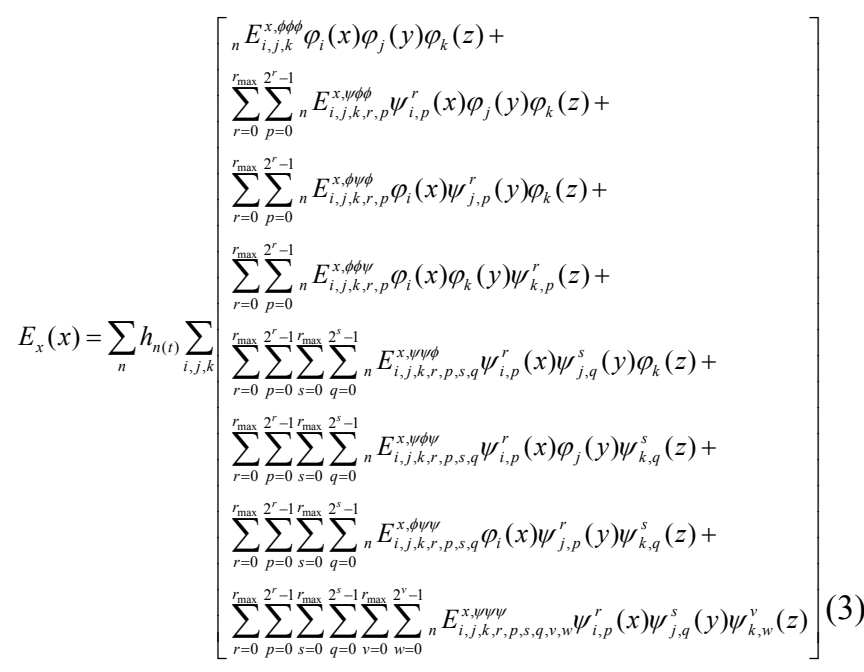

is the expansion of the $x$ component of the electric field in terms of scaling and wavelet functions. In this expression, the wavelet and scaling functions are related by (for Haar MRTD),

$$
\psi_{0}(x)=\varphi(2 x)-\varphi(2(x-1 / 2))
$$

with the scaled and translated versions of the scaling and wavelet functions defined with

$$
\begin{gathered}
\varphi_{i}(x)=\varphi\left(\frac{x}{\Delta x}-i\right), \\
\psi_{i, p}^{r}(x)=2^{r / 2} \psi_{0}\left(2^{r / 2}\left(\frac{x}{\Delta x}-i\right)-p\right) .
\end{gathered}
$$

For Haar MRTD,

$$
\varphi(x)=\chi_{[0,1)},
$$

the characteristic function between 0 and 1 (a pulse). The wavelet functions are similar, with a positive pulse for the first half of the domain and a negative pulse for the second half of the domain. In (3), it can be seen that this expansion of the fields in three dimensions takes the forms of products of all combinations of scaling and wavelet functions for all components of the fields. For $r_{\max }=0$ (one level of wavelet resolution) in $2 \mathrm{D}$, the expansion has four coefficients. These functions are presented in Fig. 1.

The offset between the fields is chosen as a function of the maximum wavelet resolution $[4,6]$. This offset is

$$
s_{d}=\frac{\Delta}{2^{r_{d, \max }+2}}
$$

where $d$ indicates a coordinate direction and indicates that the maximum resolution, and thus the offset, can depend on the direction. If coordinates of the grid are represented by a triple $(i, j, k)$, the MRTD grid can be generated by offsetting all electric field components by $s_{d}$ along their coordinate direction and offsetting the magnetic field components by $s_{d}$ along the two directions normal to their coordinate direction.
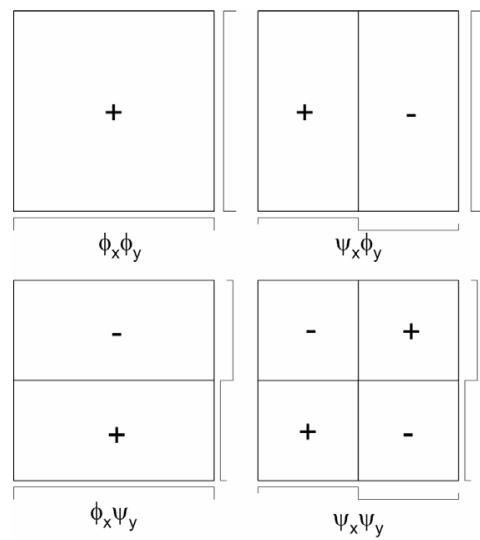

Fig. 1 Basis functions for Haar MRTD (2D) for $r_{\max }=0$

In the Haar method, when the fields are reconstructed (all wavelet/scaling coefficients are summed) they add to regions of constant value. An example of this can be seen in Fig. 1 . When the four functions are summed, the constant valued regions will have the dimensions of the four distinct regions of the $\psi_{\mathrm{x}} \psi_{\mathrm{y}}$ term. Using the offset scheme presented, the arrangement of the regions is equivalent to FDTD.

If a vector of field coefficients is defined, such as,

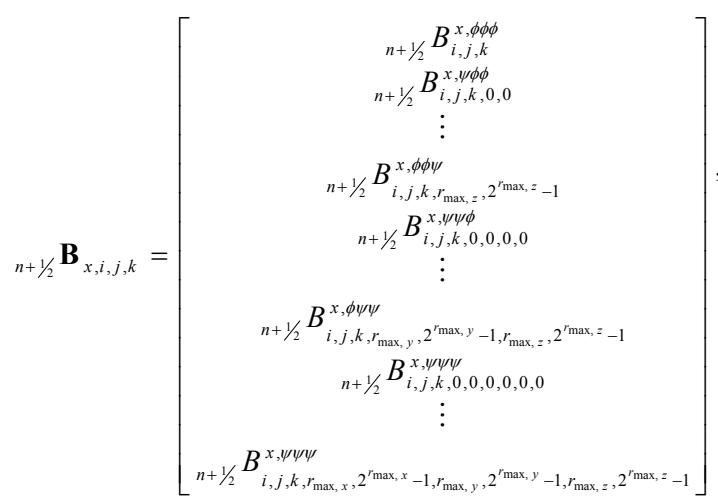

and a complementary vector of scaling/wavelet coefficients is defined, 


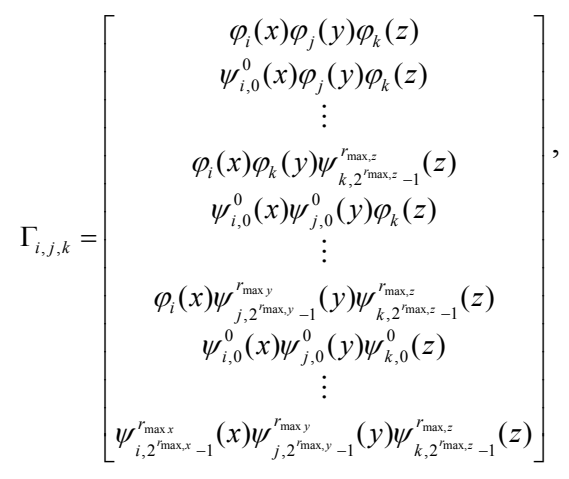

then the field expansion becomes,

$$
{ }_{n+1 / 2} B_{x}(\mathbf{r})=\sum_{i, j, k} \boldsymbol{\Gamma}_{i, j, k}^{T}{ }_{n+1 / 2} \mathbf{B}_{x, i, j, k}
$$

When the method of moments is applied to (1) using the field offset in (8), the result is

$$
\begin{aligned}
& { }_{n+1 / 2} \mathbf{B}_{x, i, j, k}={ }_{n-1 / 2} \mathbf{B}_{x, i, j, k}+ \\
& \frac{\Delta t}{\Delta x \Delta y \Delta z}\left[\sum_{m} \mathbf{U}_{E_{y}, m}^{B_{x}} \mathbf{E}_{y, i, j, k+m}+\right] . \\
& \left.\sum_{m} \mathbf{U}_{E_{z}, m n}^{B_{x}} \mathbf{E}_{z, i, j+m, k}\right]
\end{aligned}
$$

The $\mathbf{U}$ matrices are determined as

$$
\mathbf{U}_{F_{2}, m}^{F_{1}}=\left[\begin{array}{cccc}
\left\langle F_{1} \Gamma_{1},\left.\frac{\partial}{\partial n} F_{F_{2}} \Gamma_{1}\right|_{m}\right\rangle & \left\langle{ }_{F_{1}} \Gamma_{1},\left.\frac{\partial}{\partial n} F_{F_{2}} \Gamma_{2}\right|_{m}\right\rangle & \cdots & \left\langle{ }_{F_{1}} \Gamma_{1},\left.\frac{\partial}{\partial n} F_{F_{2}} \Gamma_{L}\right|_{m}\right\rangle \\
\left\langle F_{1} \Gamma_{2},\left.\frac{\partial}{\partial n} F_{2} \Gamma_{L}\right|_{m}\right\rangle & \left\langle{ }_{F_{1}} \Gamma_{2},\left.\frac{\partial}{\partial n} F_{2} \Gamma_{2}\right|_{m}\right\rangle & & \\
\vdots & \ddots & \\
\left\langle{ }_{F_{1}} \Gamma_{L},\left.\frac{\partial}{\partial n} F_{2} \Gamma_{L}\right|_{m}\right\rangle & & & \left\langle{ }_{F_{1}} \Gamma_{L},\left.\frac{\partial}{\partial n} F_{F_{2}} \Gamma_{L}\right|_{m}\right\rangle
\end{array}\right]
$$

In this case, ${ }_{F} \Gamma_{l}$ is the $l^{\text {th }}$ row of $\Gamma$ for the $F_{1}$ fields, and $\left.{ }_{F_{2}} \Gamma_{l}\right|_{m}$ is the $l^{\text {th }}$ row of $\Gamma$ for the $F_{2}$ fields, offset by $m$ in the direction of differentiation. For Haar MRTD, these matrices are only nonzero for $m=-1,0,1$ (neighboring cells). In [2] it is shown how to modify the $\mathbf{U}$ matrices to model PEC effects smaller than a cell.

\section{TIME-SPACE-ADAPTIVE GRIDDING}

In [3] a method is presented for applying a time and space adaptive grid in MRTD. This is performed through the use of two thresholds, one relative and the other absolute. The relative threshold is used to compare the wavelet functions to the scaling function to determine if they are necessary. In the Haar case, the scaling function represents the average field value across the entire cell. As the resolution increases, the sum of the Haar scaling and wavelet functions for any level represent the average over a portion of the cell for all higher levels. If the field is not rapidly varying across the cell, higher level wavelets are not necessary. By comparing the wavelet magnitude to the scaling magnitude, the contribution of the wavelet coefficient to the field magnitude can be determined.
The absolute threshold is used to determine if the wavelet coefficients are low enough to be ignored regardless of the magnitude of the scaling functions. When the field values are low (before or after the field has propagated through the grid) both the scaling and wavelet functions will be in the numerical noise level. At this point, the absolute threshold will remove unnecessary wavelet functions.

Using the two thresholds, the necessity of the wavelet functions at any time step can be determined. However, if a test is performed at each time step to decide whether to use wavelets, the scheme will not provide any efficiency (the wavelets will be calculated anyway). In order to effectively implement this technique, the thresholds must be performed at intervals of several time steps. As the time step used in MRTD is always smaller than the Nyquist limit, the time samples produced using the method represent an oversampled system. For the simulations in this paper, the wavelets were tested at intervals corresponding to the Nyquist limit.

When the wavelets are tested, the wavelets coefficients are tested for one wavelet level higher than the current resolution (if the resolution is less than $r_{\max }$ ), and the current resolution. If the wavelets at the higher resolution are significant, the resolution is increased, and likewise, if the current level wavelets are insignificant, the resolution is decreased. In this manner, the resolution can be altered on a cell by cell basis as a function of time.

\section{EXAMPLES}

The first example that is presented is that of two patch antennas printed on the same substrate separated by approximately three wavelengths. The first antenna has a resonant frequency of $31 \mathrm{GHz}$ and the second antenna has a resonant frequency of $45 \mathrm{GHz}$. The figures of merit for this structure are $S_{11}$ for each antenna and the isolation between the antennas. In addition, the simulation could be used to determine the magnitude of surface waves generated by the structure and additional components can be placed around the antennas to determine the radiation characteristics in presence of the components and the EMI/EMC of the antennas.

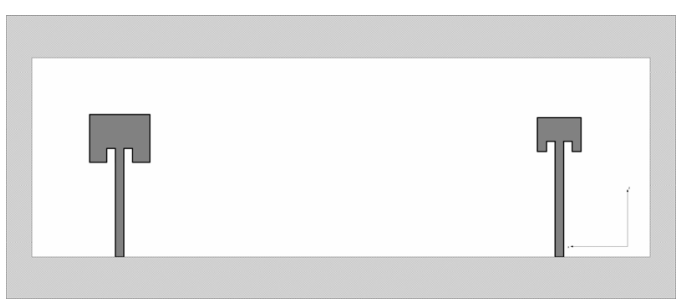

Fig. 2 Basis functions for Haar MRTD (in two dimensions) for $r_{\max }=1$

In Fig. 2 the test structure is presented. The antennas are placed on a $200 \mu \mathrm{m}$ thick substrate with $\varepsilon_{\mathrm{r}}=3.2$. The larger $(31 \mathrm{GHz})$ antenna is $3164 \mu \mathrm{m} \times 2357 \mu \mathrm{m}$ and the smaller 
antenna is $2259 \mu \mathrm{m} \times 1812.5 \mu \mathrm{m}$. Both antennas are fed by a $452 \mu \mathrm{m}$ wide $(50 \Omega)$ line.

A uniform MRTD grid was used to simulate the structure with $\Delta \mathrm{x}=452 \mu \mathrm{m}, \Delta \mathrm{y}=200 \mu \mathrm{m}, \Delta \mathrm{z}=725 \mu \mathrm{m}$, and $r_{\max }=1$ (two levels of wavelet resolution) in each direction. Both antennas showed a return loss of less than $-20 \mathrm{~dB}$ at the resonant frequency

Fig. 3 shows the number of grid points at each time step of the simulation normalized to the total number of grid points. In this case, the adaptive gridding scheme using an absolute threshold of $1 \times 10^{-5}$ of the input waveform peak and a relative threshold of $1 \times 10^{-4}$ produced a reduction of $9.4 \%$ in the number of equivalent cells (the number of basis functions required). These threshold values were chosen by trial and error; they produced an error of less that $1 \%$ in the time waveforms at the input of the antenna structures when compared to the case that did not use the adaptive grid. For the time step used in this experiment, the Nyquist limit at $50 \mathrm{GHz}$ was approximately 110 time steps.

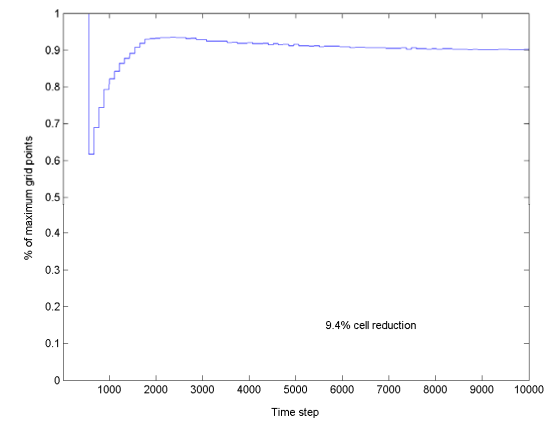

Fig. 3 Normalized number of cells for adaptive grid with initial $r=1$ everywhere

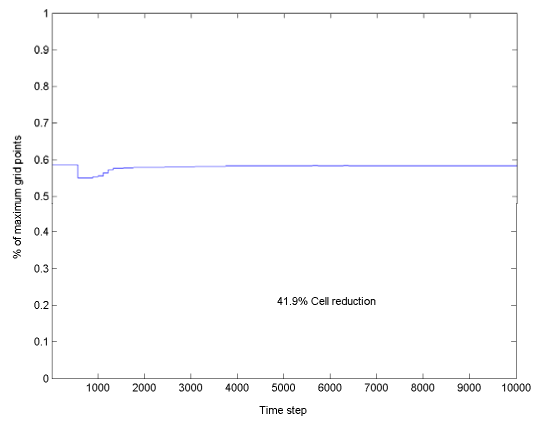

Fig. 4 Normalized number of cells for adaptive grid with fixed $\mathrm{r}=-1$ surrounding antenna

In order to improve on this efficiency, another simulation was performed where the resolution was fixed at -1 (scaling function only) surrounding the antenna ( 3 cells away from the antenna). In the antenna area, the adaptive grid was used. The results are presented in Figure 4. In this case the number of cells is reduced by $41.9 \%$.

\section{CONCLUSIONS}

For the first time, results of MRTD simulations using a time and space adaptive grid have been presented. These results show that algorithms that have been previously presented for choosing the necessary wavelet resolution as a function of time can successfully reduce the number of basis functions, and therefore execution time, necessary to model a structure. For the structure shown, the reduction in execution time is modest. In the final version of this paper, an additional example of a CPW transmission line with embedded LC components [5] will be shown. This structure has relatively small features (the gaps for the capacitors and the shunt inductor lines), surrounded by large, significantly simpler structures (the CPW lines). This is another excellent example for the technique.

There are several future avenues for this work that must be considered. While the technique that has been presented is more efficient than existing techniques, and is easy to implement, the efficiency gained is not as large as predicted for MRTD methods. In order to make the technique more efficient, other methods for determining the necessary resolution may be considered. One example is wavefront tracking, where the incident pulse is tracked through the structure. The technique presented in this paper can be used as a benchmark with which to measure more complicated methods.

\section{ACKNOWLEDGEMENT}

The authors wish to acknowledge the support of the NSF under Career Award \#9984761, the Georgia Electronic Design Center, and the Georgia Tech. NSF Packaging Research Center.

\section{REFERENCES}

[1] M. Krumpholz, L. P. B. Katehi, "New time domain schemes based on multiresolution analysis", IEEE Trans. Microwave Theory Tech., vol.44, pp.555-561, Apr. 1996.

[2] N. Bushyager and M. Tentzeris, "Composite cell MRTD for the efficient simulation of complex microwave structures," Proc. 2003 European Microwave Conference, pp. Oct. 2003.

[3] E. M. Tentzeris, A. Cangellaris, L. P. B. Katehi, and J. Harvey, "Multiresolution time-domain (MRTD) adaptive schemes using arbitrary resolutions of wavelets," IEEE Trans. Microwave Theory Tech., vol. 50, pp. 501-516, Feb. 2002.

[4] T. Dogaru and L. Carin, "Scattering analysis by the multiresolution time-domain method using compactly supported wavelet systems," IEEE Trans. Microwave Theory Tech, vol. 50, no. 7, pp.1752-1760 July 2002.

[5] A. Grbic, and G. V. Eleftheriades, "Periodic analysis of a 2-D negative refractive index transmission line structure," IEEE Trans. Antenna Prop. vol. 51, pp. 2604-2611., Oct. 2003.

[6] C. Sarris and L. P. B. Katehi, "Fundamental gridding-related dispersion effects in multiresolution time-domain schemes," IEEE Trans. Microwave Theory Tech, vol. 49, no. 12, pp 22482257, Dec. 2001. 